\title{
Advances in Gold Powder Technology
}

\section{CLOSER CON'TROL OF PARTICLE SIZE AND SHAPE}

\author{
Neville Collier \\ Johnson Matthey \& Co Limiterl, London
}

The routes for the preparation of gold poteders, required for many important industrial applications, are described in this article. Attention is drawn to the highlights of recent work designed to improve the reproducibility of particle size and shape of gold powders made by precipitalion from aqueous solutions.

The use of gold in the form of powder has increased greatly during the last decade. Probably the largest growth is associated with those powders developed for the formation of films of gold on heat resistant, usually ceramic, substrates. The films form electrically conductive paths that form the "wiring", and the bonding areas for mounting active electronics components in microcircuitry. A long established use in decorating ceramic and glass table ware, in which the visual appearance of the gold surface is the most important parameter, has also taken advantage of the new developments in gold powders.

Now that controlled particle size distribution and shape cliaracteristics, together with specifie surface properties, can be achieved there has also been a growth in the use of gold in powder metallurgy. Mass production methods in die pressing and sintering can achicve reproducibility in dimensional and weight parameters because the necessary properties for metercd flow of the powder and the packing characteristics required can be designed in to the preparation of the powder.

The powders described are in the general size group of $0.1 \mu \mathrm{m}$ to $100 \mu \mathrm{m}$ and, apart from colloidal dispersions of gold particles, this range includes almost all the gold powders used.

\section{The Importance of Reproducibility}

Reproducibility is the key to all the applications for gold powder. The knowledge that has been developed during the last few years has made it possible to precipitate gold powders in a wide range of particle size distributions and shapes with a much improved degree of reproducibility. The properties of particle size and shape, together with free flow and case of dispersion into liquids, are the limiting parameters in the three major areas of application that have been mentioned. Although they apply in powder applications with many materials other than gold, the ways in which they may be controlled have been researched and developed for gold powders following routes that are in many ways peculiar to gold.

\section{Mechanical Methods}

The major part of the devalopment of gold powders in the last few years has been concerned with the precipitation of gold in aqueous systems. It would be wrong, however, to ignore the mechanical methods of producing gold powders, some of which are very old, because these are still used in a basically unchanged form to make some powders. A particularly interesting one is based upon the craft of the gold beater and his product-gold leaf. In this process sheets of gold or a malleable gold-rich alloy are reduced in thickness by rolling and beating. Rolling the gold produces a foil between 0.001 and $0.002 \mathrm{~cm}$ thick and this is beaten in a gold beaters "book" until the thickness is reduced to something in the order of 0.2 to 0.5 um.

At this stage the edges of the leaf normally would be trimmed and the centre of the leaf packed between tissues. When the final product is to be a powder, the whole of the leaf including the thicker edges can be used. To make a powder the leaf is dispersed into a viscous, usually water soluble medium. Sugar and glucose syrups are often used. When the disperse is agitated or stirred the gold leaf is broken down into small particles. Very little welding occurs between the particles, and the powder produced in this way consists of pieces of fragmented leaf between 0.2 to $1.5 \mu \mathrm{m}$ thick.

Figure 1 shows a scanning electron micrograph of a gold powder made from gold leaf. The particle size of the powder, in terms of the diameter of a circle of area equivalent to the mean projected area of the particles, can be varied by controlling the viscosity of the medium used and typically covers the range 3 to $50 \mu \mathrm{m}$. Where a highly viscous medium is used the particles are smallcr. Dense films of gold 
Fig. 1 This powder was unade by breaking down gold lenf. The random shape of the flake-like parlicles in obvious, although there are aleo a few cryetuls prescnt $\times 625$

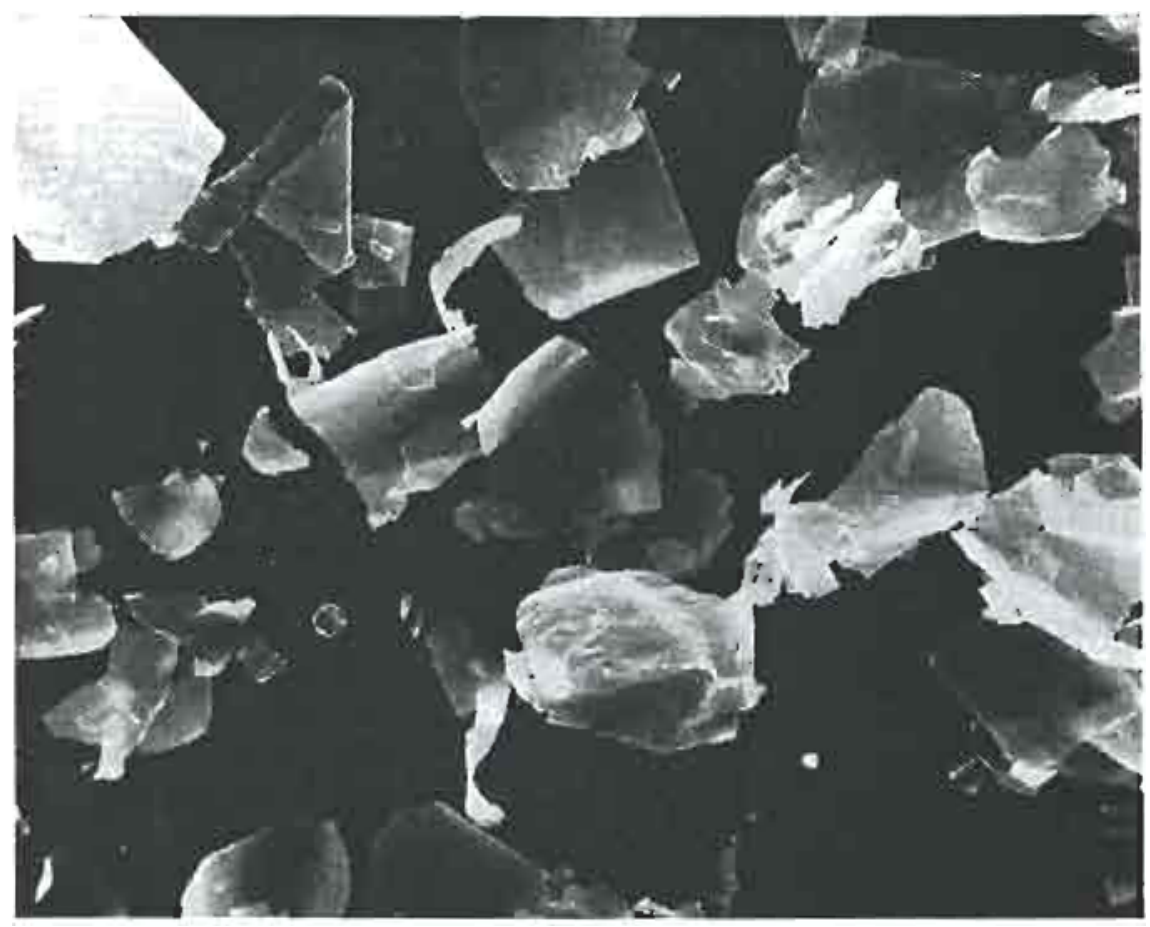

about 1 to $3 \mu \mathrm{m}$ thick can be produced by firing a film of these particles on to a variety of heat resistant substrates.

Following a method of preparation that is based almost entirely on ancient craft, the ncxt example bridges the gap in time and uses modern technology in the form of a mechanical method to modify the properties of a precipitated gold powder made by established methods. Gold powders have been produced by precipitation techniques that include ractions in aqueous systems and by the thermal decomposition of organic compounds of gold. There are problems associated with these methods that affected the quality of the products. Prior to the application of techniques that will be described later a severc degree of aggregation of the precipitated powder was often seen and little control could be exercised over particle shape. The same limitations apply to powders produced by the decomposition of organic gold compounds. In order to achieve powders with discretc particles-necessary for the development of flow characteristics for powder metallurgy and for dispersion into organic media for the preparation of elcetrically conductive paths for clectronics applications-a mechanical milling process was often used as the final stage of preparation of the powder.

Standard ball mills were used, generally with an organic solvent as a milling vehicle in which surfactants could be dissolved or dispersed in order to reduce particle-to-particle welding. In this way a proportion of the aggregates formed in the precipita- tion, washing and drying processes used to producc the gold powder could be reduced to primary particles. In addition, the packing characteristics of the powder were often improved by "rounding off the corners" of gold particles that resulted from the movement of the mill balls. Two further effccts were commonly scen. Despitc the use of surfactants, some welding was inevitable when the aggregates were deformed by the inotion of the mill balls. In addition, as very small particles werc formed by detachment from the aggregates or as a result of attrition of other particles, there was a tendency for the gold to migratc from their new high cnergy surfaces to the surfaces of larger particles. The result was a gold powder relatively frec from aggregates whose particles fittcd into a narrower particle sizc range than the original powder before milling.

A final stage in the development, prior to the control of precipitation, also involved milling, but this time in a developinent of the conventional standard ball mill.

When the internal wall of a ball mill cylinder is equipped with flanges it is possible to rotate the mill and its charge at a speed that allows the charge to be lifted to the highest point and then dropped through the mill diameter to produce an impact on the lowest point.

If a gold powder with fairly closely controlled particle size is milled in this way, the impact when the balls and powder in the milling vehicle fall causes a deformation of the powder particles to take place and produces a flakc-like powder. Flake powders made 


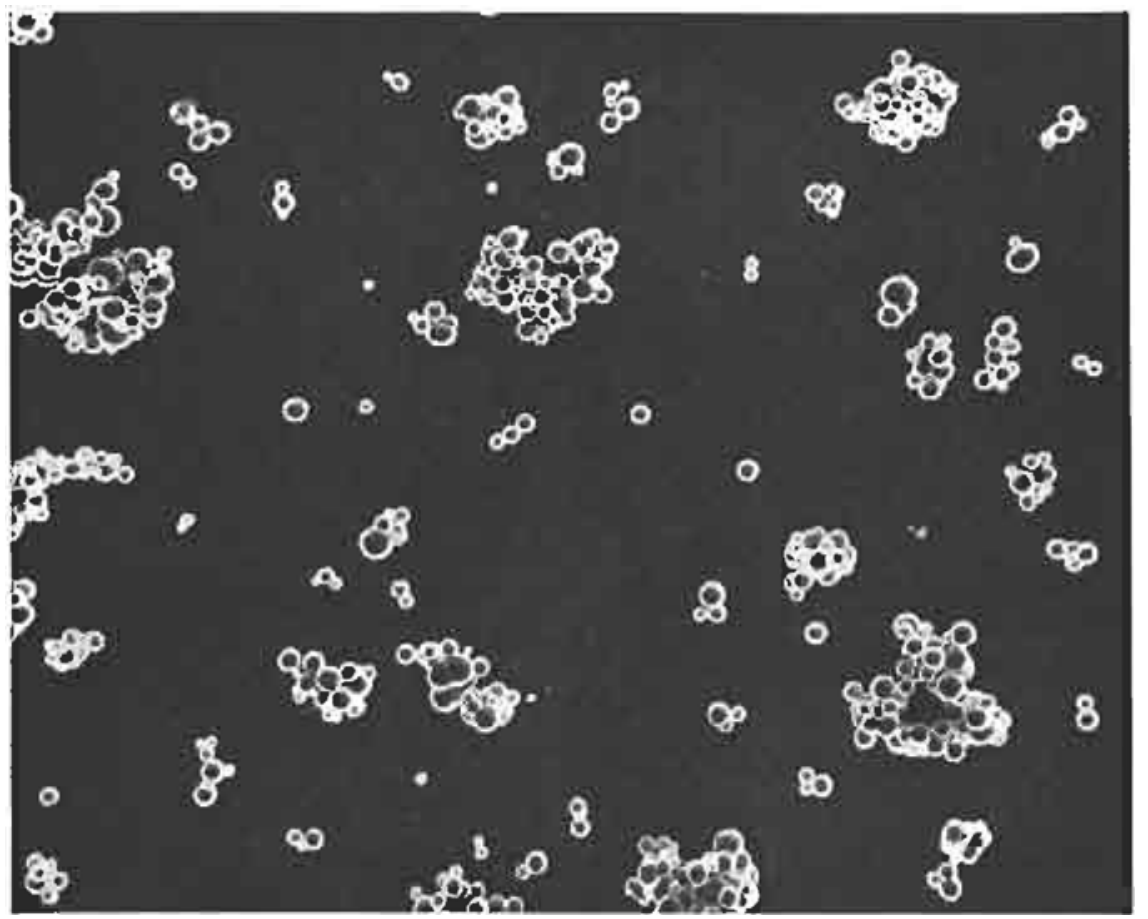

Fig. 2 The gold powder in this scanning electron micrograph illusirates tho close parlicle size limita thint en be achieved by precipilation. Virtually all tloc particles are baween $0.6 \mathrm{mul} 1.0 \mathrm{~mm}$ in size $\therefore 1250$

in this way played an important part in the development of applications for gold powder in elcctronics microcircuitry.

\section{Advances in Chemical Methods}

It is in the area of chemical reduction of gold in the form of powder from aqueous solutions that the major advances have been made in recent years. The need for such advances was presented by the growing requirements for specific properties of gold powders $(1,2,3,4)$. A summary of the ways in which the requirements have been met include the following:

The shape of gold particles can be determined by the conditions of precipitation-particularly by the type of chemical reductant used.

The use of colloidal materials during precipitation to inhibit particle to particle adhesion and growth provides control over the formation of aggrcgates.

Control of the nucleation stage followed by particle growth has made possible the precipitation of gold powders with closely controlled particle size ranges.

The formation of a discrete particle of gold in the size range 0.1 to $100 \mathrm{im}$ takes place in two stages. First the gold nucleus forms and then a period of growth follows during which quantitics of gold are deposited on the nuclcus. Exactly how a crystal nucleus is formed in a homogeneous liquid system is not known with certainty. In the preparation of gold powder the initiation of a gold nucleus is usually promoted by the use of a reducing agent. If an accurately controlled amount of reductant is dispersed very rapidly in the solution containing the gold, a reproducible number of gold nuclei will be formed. It is also possible to control the size of the

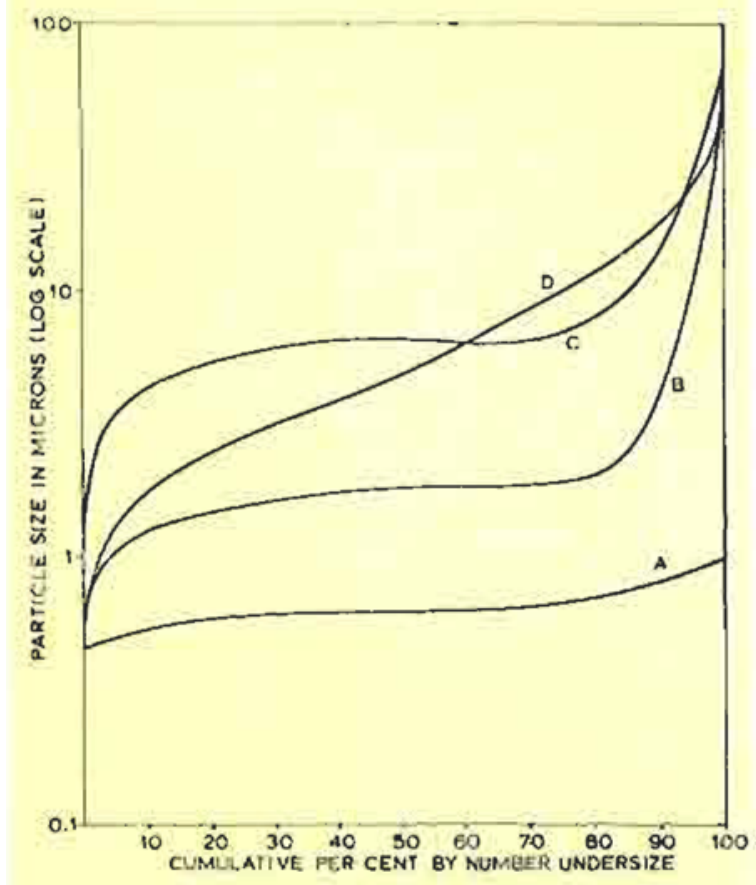

Fig. 3 Particle size distribution of four preeipitated gold powders. Many variations in particle size dintribution can be obtained by varying the number and the tinue of the uncleation ateps 
Fig. 4 The gold powder in this scanuing clectron wicrograph containg erystalline particles some of which are roughly spherical. As more sold is deposited on these parlicles they will agsume n shape similar to that of the lnrger particles $\quad \times 1250$

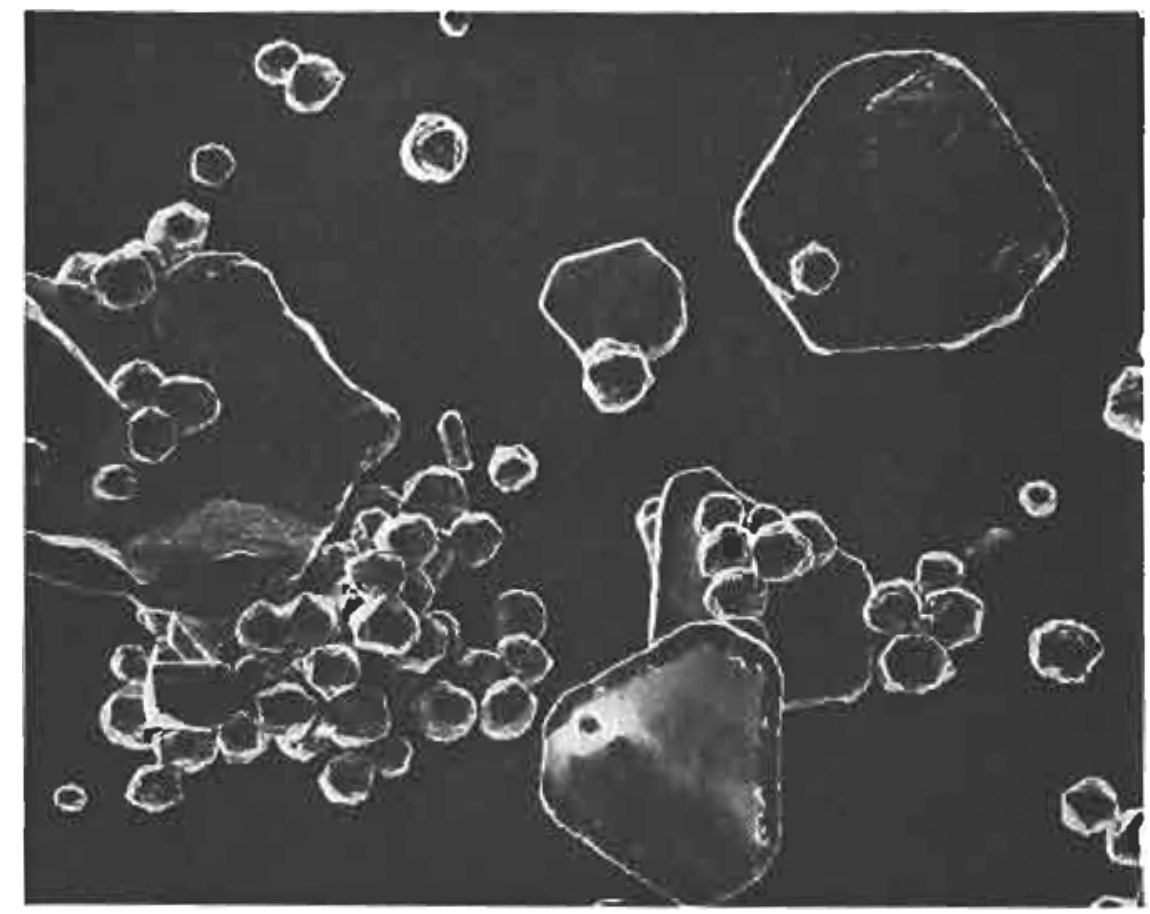

nuclei within some rather useful limits $(5,6,7)$.

When is is then required to induce growth by further deposition of gold, it is necessary to prevent flocculation of the existing nuclei. If flocculation occurs, the sites upon which the further deposition of gold takes place will be of random size and shape and the size and shape charaeteristics of the produet will reflect this starting point. The use of a lyophilic colloid will prevent both floceulation and the particle growth that is associated with the surface reaction phenomena that is characteristic of very small particles. Colloidal materials such as gelatin or gum arabic have been uscd to promote the stability of disperses of small particles. The availability of a large number of water soluble or dispersible polymeric materials has now reduced the dependence upon naturally occurring materials.

Following the preparation of a monodisperse of
Fig. 5 This is a gold powder that consists of nggregnites of omall particles and shows listle of the well formed shape characteristicf of modern powilery. It would be necesnary to uge a xuechanjeal milling process before using the powder

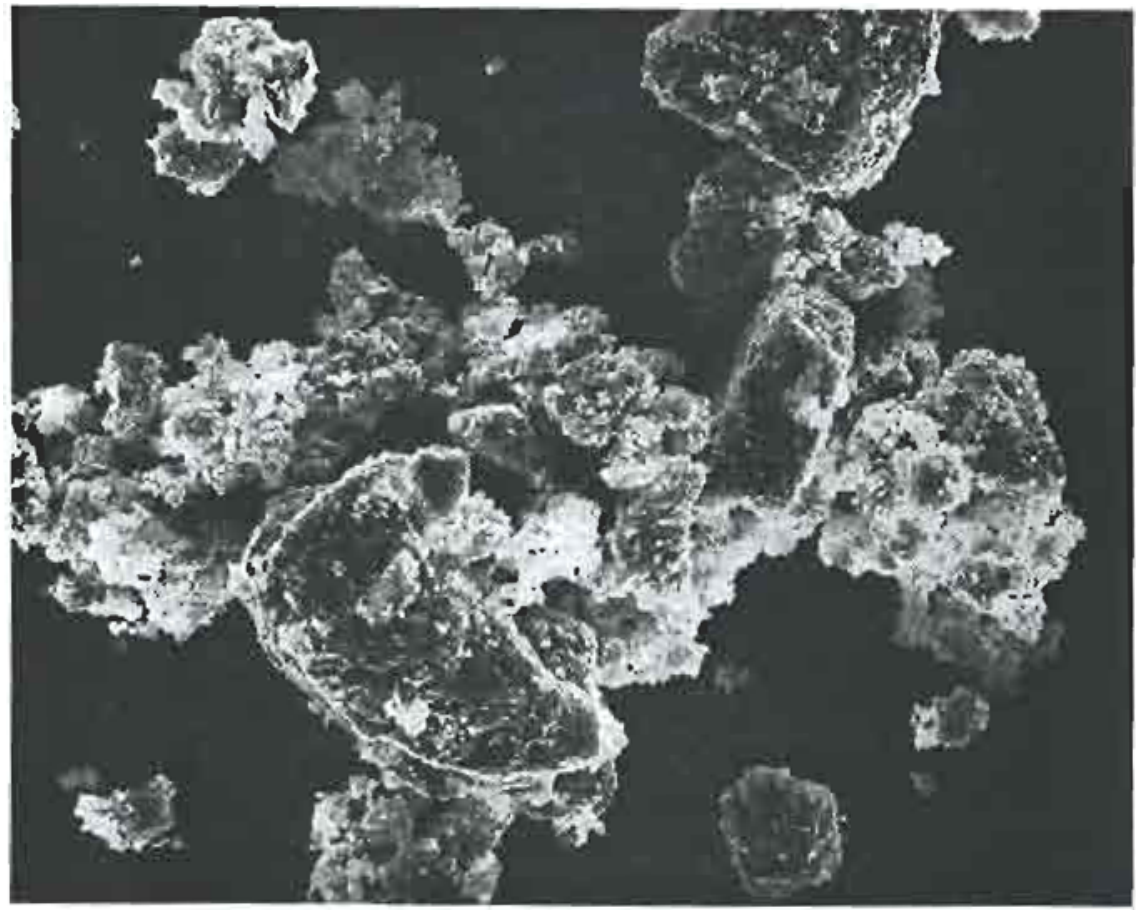


gold nuclei of the required character, the criteria being the size and number of nuclei, growth is induced by a reaction between the gold in solution and a reductant that does not easily initiate nucleation. The control parameters now include temperature and the concentration of gold in solution but two other factors have overriding influence. The first is the relationship between the number of nuclei present and the amount of available gold in solution. This determines the ultimate size of the gold particles. The second factor is the reductant used, and this can dctermine the shape and characteristics of the gold powder.

The relationship between the number of nuclei and the amount of gold available for subsequent deposition on the nuelei is simple. For a given quantity of gold the weight of each particle is proportional to the number of nuclei. The number of nuclei is reproducible by the control of temperature and concentration of reactants, and the rate of dispersion of the nucleating reductant. A typical result of the approach is shown in Figure 2, which is a scanning electron micrograph of a gold powder with a narrow particic size range similar to the plot $A$ in Figure 3. In this powder virtually all the particles are between 0.6 and $1.0 \mu \mathrm{m}$.

If more than one nucleation stage is carried out, while the growth of particles on existing nuclei is continued, then a very powerful control over particle size characteristics is exercised. By changing the number of nucleation steps, and their times during the precipitation of the powder, many variations on particle size distribution are possible. Ploc D is of a distribution similar to plot $A$, but with a much wider size range. Staggering the nucleation steps produces skewed distributions such as those of $B$ and $C$. An example of a gold powder made by using more than one nucleation stage is shown in Figure 4. The scanning electron micrograph, Figure 5, shows a typical gold powder produced by a method in which nucleation and growth were not controlled.

The shape characteristics of gold powder fall into two categorics. The gold powder particles can be almost entirely spherical. The reductants that produce spherical particles include sulphur dioxide, sulphites and related compounds.

Alternativcly the gold powder has particles that show a well defined crystallinity. This situation arises from the use of reductants covering a wide range of chemical constitution $(1,2,3,4)$.

It is clear then that the technology, upon which the preparation of a number of styles of gold powder that are important in industrial applications rests, is now at a high stage of development. As a result it is a relatively simple matter to produce gold powders, in a reproducible manner, that span a wide range of particle size distributions and fit into the broad divisions formed by spherical particle habit and flat crystalline particles.

\section{Rcferences}

1 Du Pont, U.S.A., Dutch Patent 7,114,639; 1972

2 DEMETRON, German Patcm 2,329,352; 1973

3 Du Pont, U.S.A., U.S. Patent 3,771,996; 1971

4 DEMistron, British Patem $1,420,549 ; 1974$

5 R. M. Wilenzick, D. C. Russell, R. H. Morriss and S. W. Marshall, J. Chem. Phys, 1967, 47, 533

6 G. Frens, Nature Phys. Sci, 1973, 241, (105), 20

$7 \mathrm{M}$. Nishino and E. Suito, Abs. papers, 163 rd Nat"l. Mtg., Am. Chem. Soc, 1972 (Apr.), COLLIO

\section{Ultra-high Vacuum Seal for Stratospheric Sampler COLD WELDED GOLD TUBE PROVIDES COMPLETE RELIABILITY}

When the reliability of equipment is of paramount importance it is frequently found that the unique properties of gold result in it bcing utilised for the construction of the most critical components. An interesting application for gold tubc, which makes full use of the corrosion resistance, ductility and weldability of the material has recently been reported by a group of workers at the Aeronomy Laboratory, NOAA Environmental Research Laboratories, Boulder, Colorado (Rev. Sci. Instrwm., 1976, 47, (12), 1479).

In order to collect samples of the stratosphere for analysis of chlorocarbons and other minor constituents an all-metal sampling system, which is carried aloft by a balloon and recovcred by parachute, has been developed. Prior to flight the spherical sampling containers are baked out and evacuated in the laboratory by an ultrahigh vacuum pumping unit beforc being sealed, which is done by pinching off a short length of high purity gold tubing with an external diameter of $6.35 \mathrm{~mm}$ and internal diameter $4.76 \mathrm{~mm}$. Gold is an idcal material for this pupose being capable of withstanding both the high temperature required during the baking and cvacuation stage in the laboratory and also the low temperature, about $200 \mathrm{~K}$, encountered in the stratosphere. Even after such extremes the surface of the gold is still frec from tarnish and oxidation and capable of being deformed and welded together by the modest forces available in such a lightweight system.

During the sampling and recovery stage of the flight, as the equipment descends by parachute, on-board electronic controls open the sampling container catusing it to fill with air from a pre-selected altitude and then a scaling mechanism is activated. The sealing operation is again carried out on a section of gold tube which is closed off by an clectrically fired explosive slug driving a wedge shaped bullet against one side of the tube while the other is constrained by a hardened anvil. As the inner surface of the gold tube is cold welded at the pinch-off a reliable seal is formed, and this is protected during the critical landing stage by the hardened tool steel bullet. 